\title{
MASCHKE-TYPE THEOREM FOR PARTIAL SMASH PRODUCTS
}

Liangyun Zhang and Ruifang Niu

Received: 30 March 2015; Revised: 16 July 2015

Communicated by Christian Lomp

\begin{abstract}
In this paper, we mainly study the trace function for partial Hopf actions and give a Maschke-type theorem for partial smash products.

Mathematics Subject Classification (2010): 16T05, 16S30

Keywords: Partial Hopf action, partial smash product, Maschke-type theorem
\end{abstract}

\section{Introduction}

In [10], Exel first considered partial group actions in the context of operator algebras, and studied $C^{*}$-algebras generated by partial isometries on a Hilbert space. In [6], Caenepeel and Janssen introduced partial Hopf actions regarded as a generalization of partial group actions, who was motivated by an attempt to generalize the notion of partial Galois extensions of commutative rings (see [8]), and also introduced the concept of partial smash products, which is an unital subalgebra of the usual smash products. In [12], Lomp developed the theory of partial Hopf actions, and extended the well-known results of Hopf algebras concerning smash products, such as the Blattner-Montgomery and Cohen-Montgomery theorems in [13]. Recently, the authors in [3,9] gave the Morita context between the invariant subalgebra and the partial smash product.

Let $H$ be a finite-dimensional Hopf algebra over a field $k$ and $A$ a partial $H$ module algebra. Then, the partial smash product $\underline{A \# H}$ is a ring extension of $A$, which is familiar as the partial skew group ring $A * G$ for the partial group action. In [11], the authors proved the Maschke-type theorem for the partial skew group rings. So, we naturally have the following question.

Does the Maschke-type theorem for the partial smash product $A \# H$ hold?

This work was supported by the Natural Science Foundation of China (11571173) and the Natural Science Foundation of Jiangsu Province (BK20141358). 
In this note we give a positive answer to this question by using a new method which is not just a generalization of the proof of the classical result in [7].

We always work over a fixed field $k$. Unless otherwise specified, linearity, modules and $\otimes$ are all meant over $k$. And we freely use the Hopf algebras terminology introduced in [13]. For a coalgebra $C$, we write its comultiplication $\Delta(c)=c_{1} \otimes c_{2}$, for any $c \in C$, in which we omit the summation symbols for convenience.

A partial action of the Hopf algebra $H$ on the algebra $A$ is a linear map $\alpha$ : $H \otimes A \rightarrow A$, denoted by $\alpha(h \otimes a)=h \cdot a$, for any $a, b \in A, h, g \in H$, such that

$(\mathrm{P} 1) h \cdot(a b)=\left(h_{1} \cdot a\right)\left(h_{2} \cdot b\right)$,

(P2) $1_{H} \cdot a=a$,

(P3) $h \cdot(g \cdot a)=\left(h_{1} \cdot 1_{A}\right)\left(\left(h_{2} g\right) \cdot a\right)$.

We will also call $A$ a partial $H$-module algebra. It is easy to see every action is also a partial action.

Given a Hopf algebra $H$ and a partial $H$-module algebra $A$, one can form the partial smash product $\underline{A \# H}$ which is the unital subalgebra of $A \otimes H$ defined as follows: put an algebra structure in $A \otimes H$ with the product

$$
(a \otimes h)(b \otimes k)=a\left(h_{1} \cdot b\right) \otimes h_{2} k .
$$

The partial smash product is given by

$$
\underline{A \# H}=(A \otimes H)\left(1_{A} \otimes 1_{H}\right)
$$

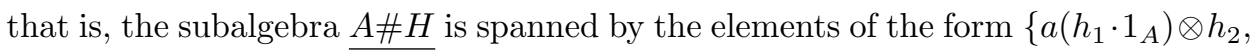
for any $a \in A, \quad h \in \overline{H\}}$. One can easily verify that the multiplication of partial smash product satisfies

$$
(\underline{a \# h}) \underline{(b \# k)}=\underline{a\left(h_{1} \cdot a\right) \# h_{2} k} .
$$

For a partial $H$-module algebra $A$ and its enveloping action $B$ given in [4], a special case which will be useful for further results is the case when $\theta(A)$ is an ideal of $H$-module algebra $B$, where the map $\theta: A \rightarrow B$ is a monomorphism of algebras. The authors in [4, Proposition 4] gave the sufficient and necessary condition, that is, for any $h, g \in H, a \in A$,

$$
h \cdot(g \cdot a)=\left(\left(h_{1} g\right) \cdot a\right)\left(h_{2} \cdot 1_{A}\right),
$$

for the element $\theta\left(1_{A}\right)$ to be a central idempotent in $B$. In our note we always assume that $A$ is an ideal of $B$, since the map $\theta: A \rightarrow B$ is a monomorphism of algebras. So, $1_{A}$ becomes a central idempotent in $B$.

Throughout this note we suppose that $H$ is always a finite dimensional Hopf algebra. 


\section{Central trace functions and invariants}

Similar to the partial group action in [11], we can define the invariants for a partial $H$-module algebra $A$ as follows:

$$
A^{H}=\left\{a \in A \mid h \cdot a=\left(h \cdot 1_{A}\right) a, \text { for any } h \in H\right\} .
$$

Note that $A^{H}$ is a subalgebra of $A$ with identity $1_{A}$. Define the trace map

$$
\hat{t}_{A}: A \rightarrow A^{H}, \quad \hat{t}_{A}(a)=t \cdot a,
$$

where $0 \neq t \in \int_{H}^{l}$ (the space of left integrals in $H$ ).

It is clear that $\hat{t}_{A}$ is a right $A^{H}$-linear map. But we hope that it is an $A^{H_{-}}$ bimodule map.

According to the references $[1,5]$, we know that lazy 1-cocycles are related with (co)homology and extensions.

A lazy 1-cocycle is a map $\ell \in \operatorname{Hom}(H, A)$ which is convolution invertible and satisfies

$$
\ell\left(h_{1}\right) \otimes h_{2}=\ell\left(h_{2}\right) \otimes h_{1},
$$

for any $h \in H$, where $A$ is a left $H$-module algebra. In particular, the unit of $\operatorname{Hom}(H, A)$, the map $h \mapsto \varepsilon(h) 1_{A}$, is a lazy 1- cocycle. If $H$ acts globally on $A$ then the unit is equal to the map $\ell(h)=h \cdot 1_{A}$.

For a partial $H$-module algebra $A$, if for any $h \in H$, the condition of lazy 1cocycles (forgetting about the condition of being convolution invertible) holds:

$$
h_{1} \cdot 1_{A} \otimes h_{2}=h_{2} \cdot 1_{A} \otimes h_{1},
$$

then, it is easy to check that $H \cdot 1_{A}$ is in $C(A)$ (the center of the algebra $A$ ), that is, for any $h \in H, a \in A$,

$$
\begin{aligned}
\left(h \cdot 1_{A}\right) a & =\left(h_{1} \cdot 1_{A}\right) \varepsilon\left(h_{2}\right) a=\left(h_{1} \cdot 1_{A}\right)\left(h_{2} S\left(h_{3}\right) \cdot a\right) \\
& =\left(h_{1} \cdot 1_{A}\right)\left(h_{2} \cdot\left(S\left(h_{3}\right) \cdot a\right)\right)=h_{1} \cdot\left(S\left(h_{2}\right) \cdot a\right) \\
& \stackrel{(2)}{=}\left(h_{1} S\left(h_{3}\right) \cdot a\right)\left(h_{2} \cdot 1_{A}\right) \stackrel{(3)}{=}\left(h_{1} S\left(h_{2}\right) \cdot a\right)\left(h_{3} \cdot 1_{A}\right) \\
& =a\left(h \cdot 1_{A}\right) .
\end{aligned}
$$

In what follows, we call the partial $H$-module algebra $A$ satisfying the equality (3) a strong partial $H$-module algebra.

\section{Remark.}

(1) The invariant subalgebra $A^{H}$ as above in this case becomes $A^{H}=\left\{a \in A \mid h \cdot a=\left(h \cdot 1_{A}\right) a=a\left(h \cdot 1_{A}\right)\right.$, for any $\left.h \in H\right\}$, see [3, Definition 5]. 
(2) If $H$ is cocommutative as coalgebra, then $A$ is a strong partial $H$-module algebra automatically.

In particular, for the partial group action, we know that it is a strong partial $H$-module algebra obviously.

(3) Let $B$ be an $H$-module algebra. Then $B$ is a trivial strong partial $H$-module algebra.

Before the next lemma we recall the definition of trace map for $H$-module algebras: let $H$ be a finite-dimensional Hopf algebra acting on an algebra $B$ with action " $\triangleright$ " and choose $0 \neq t \in \int_{H}^{l}$. Then the map $\hat{t}_{B}: B \rightarrow B^{H}$ given by $\hat{t}_{B}(b)=t \triangleright b$ is a $B^{H}$-bimodule map. We call $\hat{t}_{B}$ a (left) trace function for $H$ on $B$. From [2] we know that if $B$ is an $H$-module algebra, the surjectivity of $\hat{t}_{B}$ onto $B^{H}$ is equivalent to the existence of an element $b \in B$ with $\hat{t}_{B}(b)=1_{B}$.

In what follows, we discuss the surjectivity of trace map for a partial $H$-module algebra $A$, and throughout the rest of this section we always assume that for a partial $H$-module algebra $A$,

$$
A^{H}=\left\{a \in A \mid h \cdot a=\left(h \cdot 1_{A}\right) a=a\left(h \cdot 1_{A}\right), \text { for any } h \in H\right\} .
$$

Lemma 2.1. (1) $\hat{t}_{A}: A \rightarrow A^{H}$ is an $A^{H}$-bimodule map with values in $A^{H}$.

Let $(B, \theta)$ be an enveloping action of a partial $H$-module algebra $A$. Then

(2) $\hat{t}_{A}(a)=\hat{t}_{B}(a) 1_{A}$, for any $a \in A$;

(3) $\hat{t}_{B}(B)=\hat{t}_{B}(A)$.

Proof. (1) For any $a \in A, c \in A^{H}$, we have

$$
\begin{aligned}
c \hat{t}_{A}(a) & =c(t \cdot a)=c\left(t_{1} \cdot 1_{A}\right)\left(t_{2} \cdot a\right) \\
& =\left(t_{1} \cdot c\right)\left(t_{2} \cdot a\right)=t \cdot(c a)=\hat{t}_{A}(c a), \\
\hat{t}_{A}(a) c & =(t \cdot a) c=\left(t_{1} \cdot a\right)\left(t_{2} \cdot 1_{A}\right) c \\
& =\left(t_{1} \cdot a\right)\left(t_{2} \cdot c\right)=t \cdot(a c)=\hat{t}_{A}(a c) .
\end{aligned}
$$

(2) It is obvious from [4, Proposition 1].

(3) We only show that $\hat{t}_{B}(B) \subseteq \hat{t}_{B}(A)$, the opposite is obvious. Assume that there exists an element $x \in B$ such that $\hat{t}_{B}(x)=b \in \hat{t}_{B}(B)$, where the element $x$ is of the form $\Sigma_{i} h_{i} \triangleright a_{i}$, for a finite number of elements $h_{i} \in H, a_{i} \in A$. Then

$$
b=\hat{t}_{B}(x)=t \triangleright\left(\Sigma_{i} h_{i} \triangleright a_{i}\right)=\Sigma\left(t h_{i}\right) \triangleright a_{i} \in \hat{t}_{B}(A),
$$

so $\hat{t}_{B}(B)=\hat{t}_{B}(A)$.

Proposition 2.2. (1) $\hat{t}_{A}$ is onto $A^{H}$ if and only if there exists an element $a \in A$ such that $\hat{t}_{A}(a)=1_{A}$. 
(2) Assume that $(B, \theta)$ is an enveloping action of a partial $H$-module algebra A. If $\hat{t}_{B}$ is onto $B^{H}$, then $\hat{t}_{A}$ is onto $A^{H}$.

Proof. (1) Let there exist an element $a \in A$ such that $\hat{t}_{A}(a)=1_{A}$. Then, for any $c \in A^{H}, c=c 1_{A}=c \hat{t}_{A}(a)=\hat{t}_{A}(c a)$, that is, $\hat{t}_{A}$ is onto $A^{H}$. Conversely, it is straightforward.

(2) If there is an element $b \in B$ with $\hat{t}_{B}(b)=1_{B}$, then, by Lemma 2.1 , there exists an element $a \in A$ such that $\hat{t}_{B}(a)=1_{B}$. So, the fact that $h \cdot a=1_{A}(h \triangleright a)=(h \triangleright a) 1_{A}$ implies $\hat{t}_{A}(a)=\hat{t}_{B}(a) 1_{A}=1_{A}$. According to the above conclusion, we know that $\hat{t}_{A}$ is onto $A^{H}$.

\section{Maschke-type theorem for partial smash products}

In this section, we assume that $A$ is a strong partial $H$-module algebra, and give the Maschke-type theorem for partial smash product by using a kind of new method.

Lemma 3.1. In partial smash product $\underline{A \# H}$ : for any $a \in A, h \in H$,

$$
\underline{a \# h}=\left(\underline{1_{A} \# h_{2}}\right)\left(S^{-1}\left(h_{1}\right) \cdot a \# 1_{H}\right) .
$$

Proof. For any $a \in A, \quad h \in H$, we have

$$
\begin{aligned}
\underline{a \# h} & =a\left(h_{1} \cdot 1_{A}\right) \# h_{2}=\left(\left(h_{2} S^{-1}\left(h_{1}\right)\right) \cdot a\right)\left(h_{3} \cdot 1_{A}\right) \# h_{4} \\
& \stackrel{(2)}{=} h_{2} \cdot\left(S^{-1}\left(h_{1}\right) \cdot a\right) \# h_{3}=\left(h_{2} \cdot 1_{A}\right)\left(h_{3} \cdot\left(S^{-1}\left(h_{1}\right) \cdot a\right)\right) \# h_{4} \\
& =\left(h_{2} \cdot 1_{A} \# h_{3}\right)\left(S^{-1}\left(h_{1}\right) \cdot a \# 1_{H}\right) \\
& =\left(\underline{1_{A} \# h_{2}}\right)\left(S^{-1}\left(h_{1}\right) \cdot a \# 1_{H}\right) .
\end{aligned}
$$

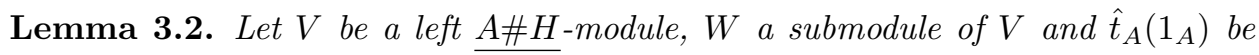
invertible in $A$. Assume that $\lambda: V \rightarrow W$ is a projection as $A$-modules. Then, there

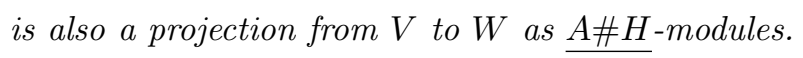

Proof. Assume that $\lambda: V \rightarrow W$ be the projection as $A$-modules. Define the map

$$
\widetilde{\lambda}: V \rightarrow W \text { by } \widetilde{\lambda}(v)=u\left(\underline{1_{A} \# S\left(x_{1}\right)}\right) \lambda\left(\left(\underline{1_{A} \# x_{2}}\right) v\right),
$$

where $u=\left(\hat{t}_{A}\left(1_{A}\right)\right)^{-1}, S(x)=t$, as in Section 2, $\hat{t}_{A}\left(1_{A}\right)=t \cdot 1_{A}, 0 \neq t \in \int_{H}^{l}, x \in$ $\int_{H}^{r}$. 


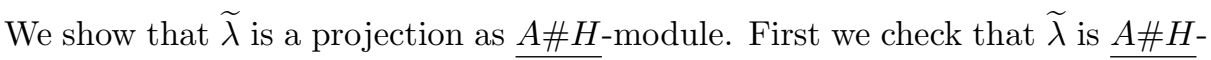
linear. Since $S$ is bijective, we can choose $a \# S(h) \in A \# H$ :

$$
\begin{aligned}
& u^{-1}(a \# S(h)) \widetilde{\lambda}(v) \\
& =u^{-1}(a \# S(h)) u\left(1_{A} \# S\left(x_{1}\right)\right) \lambda\left(\left(1_{A} \# x_{2}\right) v\right)
\end{aligned}
$$

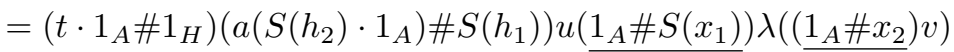

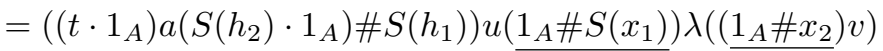

$$
\begin{aligned}
& =\left(a\left(S\left(h_{2}\right) \cdot 1_{A}\right)\left(t \cdot 1_{A}\right) \# S\left(h_{1}\right)\right) u\left(1_{A} \# S\left(x_{1}\right)\right) \lambda\left(\left(1_{A} \# x_{2}\right) v\right) \\
& =\left(a\left(S\left(h_{3}\right) \cdot 1_{A}\right)\left(S\left(h_{2}\right) t \cdot 1_{A}\right) \# S\left(h_{1}\right)\right) u\left(1_{A} \# S\left(x_{1}\right)\right) \lambda\left(\left(1_{A} \# x_{2}\right) v\right)
\end{aligned}
$$

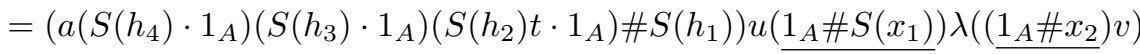

$$
\begin{aligned}
& \stackrel{(2)}{=}\left(a\left(S\left(h_{3}\right) \cdot 1_{A}\right)\left(S\left(h_{2}\right) \cdot\left(t \cdot 1_{A}\right)\right) \# S\left(h_{1}\right)\right) u\left(1_{A} \# S\left(x_{1}\right)\right) \lambda\left(\left(1_{A} \# x_{2}\right) v\right) \\
& \left.=\left(a\left(S\left(h_{2}\right) \cdot 1_{A}\right) \# S\left(h_{1}\right)\right)\left(t \cdot 1_{A} \# 1_{H}\right) u\left(\underline{1_{A} \# S\left(x_{1}\right)}\right) \lambda\left(\underline{\left(1_{A} \# x_{2}\right.}\right) v\right) \\
& =\left(\underline { a \# S ( h ) ) } \left(\underline{\left.1_{A} \# S\left(x_{1}\right)\right)} \lambda\left(\left(\underline{1_{A} \# x_{2}}\right) v\right)\right.\right. \\
& \stackrel{(1)}{=}\left(a\left(S\left(h_{2}\right) \cdot 1_{A}\right) \# S\left(x_{1} h_{1}\right)\right) \lambda\left(\left(1_{A} \# x_{2}\right) v\right) \\
& \stackrel{(4)}{=}\left(1_{A} \# S\left(x_{1} h_{1}\right)\right)\left(S^{-1}\left(S\left(x_{2} h_{2}\right)\right) \cdot\left(a\left(S\left(h_{3}\right) \cdot 1_{A}\right)\right) \# 1_{H}\right) \lambda\left(\left(1_{A} \# x_{3}\right) v\right) \\
& =\left(\underline{\left.1_{A} \# S\left(x_{1} h_{1}\right)\right)}\right)\left(x_{2} h_{2} \cdot\left(a\left(S\left(h_{3}\right) \cdot 1_{A}\right)\right) \# 1_{H}\right) \lambda\left(\left(\underline{\left.1_{A} \# x_{3}\right)} v\right)\right. \\
& =\left(\underline{\left.1_{A} \# S\left(x_{1} h_{1}\right)\right)} \lambda\left(\underline{\left(x_{2} h_{2} \cdot\left(a\left(S\left(h_{3}\right) \cdot 1_{A}\right)\right) \# x_{3}\right)} v\right)\right. \\
& =\left(\underline { 1 _ { A } \# S ( x _ { 1 } h _ { 1 } ) ) } \lambda \left(\left(\underline{\left.\left.\left.x_{2} h_{2} \cdot a\right)\left(x_{3} h_{3} \cdot\left(S\left(h_{4}\right) \cdot 1_{A}\right)\right) \# x_{4}\right) v\right)}\right.\right.\right. \\
& =\left(1_{A} \# S\left(x_{1} h_{1}\right)\right) \lambda\left(\left(\left(x_{2} h_{2} \cdot a\right)\left(x_{3} h_{3} S\left(h_{4}\right) \cdot 1_{A}\right) \# x_{4}\right) v\right) \\
& =\left(\underline{\left.1_{A} \# S\left(x_{1} h_{1}\right)\right)}\right) \lambda\left(\left(\left(x_{2} h_{2} \cdot a\right)\left(x_{3} \cdot 1_{A}\right) \# x_{4}\right) v\right) \\
& =\left(1_{A} \# S\left(x_{1} h_{1}\right)\right) \lambda\left(\left(x_{2} h_{2} \cdot a \# x_{3}\right) v\right) \text {. }
\end{aligned}
$$

Since $x$ is a right integral in $H$, we have

$$
\begin{aligned}
x_{1} h_{1} \otimes x_{2} h_{2} \otimes x_{3} & =\left((\Delta \otimes i d) \Delta\left(x h_{1}\right)\right)\left(1_{H} \otimes 1_{H} \otimes S\left(h_{2}\right)\right) \\
& =((\Delta \otimes i d) \Delta(x))\left(1_{H} \otimes 1_{H} \otimes S(h)\right) \\
& =x_{1} \otimes x_{2} \otimes x_{3} S(h) .
\end{aligned}
$$

Now we use above equation to compute:

$$
\begin{aligned}
(5) & =\left(\underline{\left.1_{A} \# S\left(x_{1}\right)\right)} \lambda\left(\underline{\left(x_{2} \cdot a \# x_{3} S(h)\right)} v\right)\right. \\
& \stackrel{(1)}{=}\left(\underline { 1 _ { A } \# S ( x _ { 1 } ) ) } \lambda \left(\underline{\left.\left(\left(1_{A} \# x_{2}\right)(a \# S(h))\right) v\right)}\right.\right. \\
& =u^{-1} u\left(\underline{1_{A} \# S\left(x_{1}\right)}\right) \lambda\left(\left(\left(\underline{\left.1_{A} \# x_{2}\right)}\right)(\underline{a \# S(h))}) v\right)\right. \\
& =u^{-1} \widetilde{\lambda}(\underline{(a \# S(h))} v),
\end{aligned}
$$

so $\widetilde{\lambda}$ is $A \# H$-linear. From the above computation, we conclude that

$$
(\underline{a \# S(h)})\left(\underline{1_{A} \# S\left(x_{1}\right)}\right) \otimes_{A}\left(\underline{1_{A} \# x_{2}}\right)=\left(\underline{1_{A} \# S\left(x_{1}\right)}\right) \otimes_{A}\left(\underline{1_{A} \# x_{2}}\right)(\underline{a \# S(h)}) .
$$


It remains to check that $\tilde{\lambda}$ is a projection. If $w \in W$, then we have

$$
\begin{aligned}
\tilde{\lambda}(w) & =u\left(\underline{1_{A} \# S\left(x_{1}\right)}\right)\left(\underline{1_{A} \# x_{2}}\right) w=u\left(\underline{S\left(x_{2}\right) \cdot 1_{A} \# S\left(x_{1}\right) x_{3}}\right) w \\
& \stackrel{(3)}{=} u\left(\underline{\left(x_{1}\right) \cdot 1_{A} \# S\left(x_{2}\right) x_{3}}\right) w=u\left(S(x) \cdot 1_{A} \# 1_{H}\right) w \\
& =u\left(S(x) \cdot 1_{A}\right) w=u\left(t \cdot 1_{A}\right) w=w .
\end{aligned}
$$

According to Lemma 3.2, we get the following main result.

Theorem 3.3. Under the same assumptions as above. If A is semisimple Artinian, then $\underline{A \# H}$ is semisimple Artinian.

Remark. Since $H$ is not a subalgebra of the partial smash product $A \# H$, from the proof of Lemma 3.2, we can see that we use a new method which is not just a generalization of the proof of the classical result in [7] to prove the Maschke-type theorem.

Note that an $H$-module algebra $B$ is a trivial strong partial $H$-module algebra, $H$ is semisimple iff $\varepsilon(t) \neq 0$, where $0 \neq t \in \int_{H}^{l}$, and $\hat{t}_{B}\left(1_{B}\right)=t \triangleright 1_{B}=\varepsilon(t) 1_{B}$ is invertible in $B$ iff $\varepsilon(t) \neq 0$. So, in this case the semisimplity of $H$ is equivalent to the invertibility of $\hat{t}_{B}\left(1_{B}\right)$ in $B$. What's more, the partial smash product $\underline{A \# H}$ become a partial skew group ring $A \star_{\alpha} G$ in case of replacing $H$ by $k G$. Therefore, we have the following results.

Corollary 3.4. Let $H$ be a finite-dimensional semisimple Hopf algebra, and $B$ an $H$-module algebra. If $B$ is semisimple, then $B \# H$ is semisimple.

The above corollary is a generalization of Theorem 6 in [7].

Corollary 3.5. Let $\alpha$ be a partial action of a finite group $G$ on a unital algebra $R$. If $R$ is semisimple and $\hat{t}_{R}\left(1_{R}\right)$ is invertible in $R$, then the partial skew group ring $R \star_{\alpha} G$ is semisimple.

The above corollary is a generalization of Corollary 3.3 in [11].

In what follows, we consider the separability of $\underline{A \# H}$ under the condition that $\hat{t}_{A}\left(1_{A}\right)$ is invertible in $A$.

Proposition 3.6. Assume that $\hat{t}_{A}\left(1_{A}\right)$ is invertible in $A$. Then $\underline{A \# H}$ is separable over $A$.

Proof. As in Section 2, let $\hat{t}_{A}\left(1_{A}\right)=t \cdot 1_{A}$ be invertible in $A$ with the inverse $u$. It is easy to prove $u \in C(A)$. Moreover, for any $h \in H$,

$$
\begin{aligned}
h \cdot u-\left(h \cdot 1_{A}\right) u & =\left(h \cdot u-\left(h \cdot 1_{A}\right) u\right)\left(t \cdot 1_{A}\right) u=(h \cdot u)\left(t \cdot 1_{A}\right) u-\left(h \cdot 1_{A}\right) u \\
& =\left(h_{1} \cdot u\right)\left(h_{2} t \cdot 1_{A}\right) u-\left(h \cdot 1_{A}\right) u=\left(h \cdot\left(u\left(t \cdot 1_{A}\right)\right)\right) u-\left(h \cdot 1_{A}\right) u \\
& =\left(h \cdot 1_{A}\right) u-\left(h \cdot 1_{A}\right) u=0
\end{aligned}
$$


shows $u \in A^{H}$. Hence $u \in C(A) \cap A^{H}$. Consider the element

$$
w=\left(\underline{1_{A} \# t_{2}}\right) \otimes_{A}\left(\underline{u \# S^{-1}\left(t_{1}\right)}\right) \in \underline{A \# H} \otimes_{A} \underline{A \# H} .
$$

In the following, we will show that $w$ is a separability idempotent for $A \# H$.

Let $\mu: \underline{A \# H} \otimes_{A} \underline{A \# H} \rightarrow \underline{A \# H}$ denote the multiplication map. Then

$$
\begin{aligned}
\mu(w) & =\underline{\left(1_{A} \# t_{2}\right)}\left(u \# S^{-1}\left(t_{1}\right)\right)=\underline{t_{2} \cdot u \# t_{3} S^{-1}\left(t_{1}\right)} \\
& =\underline{\left(t_{2} \cdot 1_{A}\right) u \# t_{3} S^{-1}\left(t_{1}\right)}=\underline{\left(t_{1} \cdot 1_{A}\right) u \# t_{3} S^{-1}\left(t_{2}\right)} \\
& =\underline{\left(t \cdot 1_{A}\right) u \# 1_{H}=1_{A} \# 1_{H} .}
\end{aligned}
$$

As in Lemma 3.2, we choose $S(x)=t$, where $0 \neq t \in \int_{H}^{l}, x \in \int_{H}^{r}$, and choose $a \# S(h) \in \underline{A \# H}$,

$$
\begin{aligned}
\underline{(a \# S(h)) w} & =\underline{(a \# S(h))}\left(\underline{1_{A} \# t_{2}}\right) \otimes_{A}\left(\underline{u \# S^{-1}\left(t_{1}\right)}\right) \\
& =\underline{(a \# S(h)})\left(\underline { 1 _ { A } \# S ( x _ { 1 } ) ) } \otimes _ { A } \left(\underline{\left.u \# x_{2}\right)}\right.\right. \\
& =\underline{(a \# S(h)})\left(\underline{1_{A} \# S\left(x_{1}\right)}\right) \otimes_{A} \underline{u\left(\underline{1_{A} \# x_{2}}\right)} \\
& \left.\left.\stackrel{(6)}{=} \underline{\left(1_{A} \# S\left(x_{1}\right)\right.}\right) \otimes_{A} u\left(\underline{1_{A} \# x_{2}}\right) \underline{(a \# S(h)}\right) \\
& \left.=\underline{\left(1_{A} \# S\left(x_{1}\right)\right.}\right) \otimes_{A}\left(\underline{\left.u \# x_{2}\right)}\right) \underline{(a \# S(h))} \\
& \left.=\underline{\left(1_{A} \# t_{2}\right)} \otimes_{A}\left(\underline{u \# S^{-1}\left(t_{1}\right)}\right) \underline{(a \# S(h)}\right) \\
& =w \underline{(a \# S(h)}),
\end{aligned}
$$

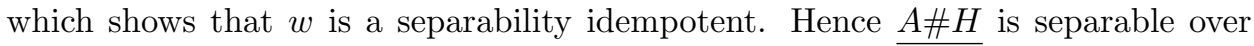
A.

Question. In [3], the authors defined the partial invariants $A^{H}=\{a \in A \mid h \cdot a=$ $\left(h \cdot 1_{A}\right) a=a\left(h \cdot 1_{A}\right)$, for any $\left.h \in H\right\}$, and gave the Morita context between the invariant subalgebra $A^{H}$ and the partial smash product $A \# H$. In our note, we introduce the condition (3) of lazy 1-cocycles related with cohomology and extensions in order to prove the Maschke-type theorem. We hope that this condition in the future can be improved.

Acknowledgment. The authors would like to thank the referee for the valuable suggestions and comments.

\section{References}

[1] A. L. Agore and G. Militaru, Extending structures II: the quantum version, J. Algebra, 336 (2011), 321-341.

[2] E. Aljadeff, On the surjectivity of some trace maps, Israel J. Math., 86 (1994), 221-232. 
[3] M. M. S. Alves and E. Batista, Partial Hopf actions, partial invariants and a Morita context, Algebra Discrete Math., 3 (2009), 1-19.

[4] M. M. S. Alves and E. Batista, Enveloping actions for partial Hopf actions, Comm. Algebra, 38(8) (2010), 2872-2902.

[5] J. Bichon and C. Kassel, The lazy homology of a Hopf algebra, J. Algebra, 323(9) (2010), 2556-2590.

[6] S. Caenepeel and K. Janssen, Partial (co)actions of Hopf algebras and partial Hopf-Galois theory, Comm. Algebra, 36(8) (2008), 2923-2946.

[7] M. Cohen and D. Fishman, Hopf algebra actions, J. Algebra, 100(2) (1986), 363-379.

[8] M. Dokuchaev, M. Ferrero and A. Paques, Partial actions and Galois theory, J. Pure Appl. Algebra, 208(1) (2007), 77-87.

[9] C. Z. Du and J. F. Lin, Morita context, partial Hopf Galois extensions and partial entwining structure, Math. Notes, 95 (2014), 43-52.

[10] R. Exel, Circle actions on $C^{*}$-algebras, partial automorphisms and generalized Pimsner-Voiculescu exact sequences, J. Funct. Anal., 122(2) (1994), 361-401.

[11] M. Ferrero and J. Lazzarin, Partial actions and partial skew group rings, J. Algebra, 319(12) (2008), 5247-5264.

[12] C. Lomp, Duality for partial group actions, Int. Electron. J. Algebra, 4 (2008), $53-62$.

[13] S. Montgomery, Hopf Algebras and Their Actions on Rings, CBMS Regional Conference Series in Mathematics, 82, Published for the Conference Board of the Mathematical Sciences, Washington, DC; by the Amer. Math. Soc., Providence, RI, 1993.

\author{
Liangyun Zhang and Ruifang Niu \\ Nanjing Agricultural University \\ 210095 Nanjing, China \\ e-mail: zlyun@njau.edu.cn (L. Y. Zhang)
}

